\title{
Efecto de la relación calcio y fósforo sobre las características óseas, porcentaje de cenizas e integridad esquelética en pollos de carne
}

\author{
Effect of the calcium phosphorus ratio on bone characteristics, percent of ashes \\ and skeletal integrity of broilers
}

Cristian Uculmana M. ${ }^{1,3}$, Diego Martínez-Patiño², Otto Zea M. ${ }^{1}$, Carlos Vílchez P. ${ }^{1}$

\section{RESUMEN}

\begin{abstract}
El objetivo del presente ensayo fue determinar el efecto de la relación calcio $(\mathrm{Ca})$ y fósforo disponible $(\mathrm{Pd})$ sobre la morfometría y mineralización ósea e integridad esquelética de pollos de carne. Se utilizaron 60 pollos de la línea Cobb 500 de un día de edad distribuidos en 12 unidades experimentales y asignados, durante 21 días, a uno de los siguientes tratamientos: T1, Ca:Pd baja (1.13); T2, Ca:Pd media (1.55); T3, Ca:Pd adecuada (1.98). Todas las dietas contenían $3072 \mathrm{Kcal}$ de EM/kg y $20.50 \%$ de proteína cruda. El alimento, en forma de harina, y el agua fue ofrecido ad libitum. En el día 21 de edad se sacrificaron todos los animales para extraer fémures, tibias y metatarsos. Se evaluaron variables que incluyeron morfometría ósea (peso, longitud, ancho, volumen y densidad), integridad esquelética (capacidad para caminar y lesiones en la cabeza femoral) y se determinó el contenido de ceniza en la tibia. La data fue analizada empleando un Diseño Completamente al Azar usando el procedimiento ANOVA y la prueba de Duncan para la comparación de medias. Los resultados mostraron que el contenido de ceniza en tibia fue afectado significativamente $(\mathrm{p}<0.01)$ por cada cambio en la relación $\mathrm{Ca}: \mathrm{Pd}$. En comparación con las relaciones $\mathrm{Ca}: \mathrm{Pd}$ de 1.98 y 1.55, la relación $\mathrm{Ca}: \mathrm{Pd}$ de 1.13 afectó la capacidad para caminar $(p<0.03)$ y la densidad de la tibia $(p<0.05)$. En conclusión, el contenido de ceniza en tibia es sensible a cambios en la relación $\mathrm{Ca}: \mathrm{Pd}$, mientras que indicadores de integridad esquelética solo son afectados cuando la relación Ca:Pd está por debajo de 1.55.
\end{abstract}

Palabras clave: calcio; fósforo; morfometría ósea; pollos de engorde

\footnotetext{
${ }^{1}$ Departamento Académico de Nutrición, Facultad de Zootecnia, Universidad Nacional Agraria La Molina, Lima, Perú

${ }^{2}$ LIAN Desarrollo y Servicio S.A.C., Lima, Perú

${ }^{3}$ E-mail: cristian.uculmana@gmail.com
}

Recibido: 2 de abril de 2018

Aceptado para publicación: 4 de septiembre de 2018 
The aim of this study was to determine the effect of the ratio calcium $(\mathrm{Ca})$ and available phosphorus (AP) on the bone morphometry and mineralization, and skeletal integrity of broilers. Sixty one day-old Cobb 500 chicks were distributed in 12 experimental units and assigned, for 21 days, to one of the following treatments: T1, low Ca:Pd (1.13); T2, medium Ca:Pd (1.55); T3, adequate Ca:Pd (1.98). All diets contained $3072 \mathrm{Kcal}$ $\mathrm{ME} / \mathrm{kg}$ and $20.50 \%$ crude protein. Feeds, in meal form, and water were offered ad libitum. At day 21 of age all animals were slaughtered to extract the femur, tibia and metatarsus. Variables that included bone morphometry (weight, length, width, volume and density), skeletal integrity (ability to walk and lesions in the femoral head) were measured and the content of ash in the tibia was determined. The data was analyzed under a Complete Randomized Design using the ANOVA procedure and Duncan Test for comparison of the means. The results showed that the content of ash in tibia was significantly affected $(\mathrm{p}<0.01)$ by the dietary $\mathrm{Ca}: \mathrm{Pd}$ ratio. Compared with the 1.98 and $1.55 \mathrm{Ca}: \mathrm{Pd}$ ratios, the 1.13 $\mathrm{Ca}: \mathrm{Pd}$ ratio affected $(\mathrm{p}<0.03)$ the capacity to walk and the density of the tibia $(\mathrm{p}<0.05)$. In conclusion, the ash content in tibia is sensitive to changes in the $\mathrm{Ca}: \mathrm{Pd}$ ratios while skeletal integrity indicators are only affected when the Ca:Pd ratio was lower than1.55.

Key words: calcium; phosphorus; bone morphometry; broilers

\section{INTRODUCCIÓN}

El progresivo desarrollo del sector avícola trajo consigo una mayor susceptibilidad a desórdenes esqueléticos, particularmente en la pierna, debidos al rápido crecimiento de los pollos de engorde, predisponiendo la rotura de huesos durante el proceso de captura y el transporte (Kayode et al., 2016) y reduciéndose así la productividad de las aves y aumentando la mortalidad (Han et al., 2015), generando pérdidas económicas.

Diversos factores son conocidos de afectar la mineralización ósea en pollos de carne, entre los que se encuentran enfermedades, genética, sistema endocrino, carga física, sexo, crecimiento, toxinas, antinutrientes y la nutrición (Rath et al., 2000). La relación con el factor nutrición está en el contenido de calcio y el fósforo en la dieta, que es la que determina la mineralización y dureza adecuada del hueso. Así, por ejemplo, el 99\% del contenido de calcio (Ca) en el cuerpo forma parte de la estructura del esqueleto. El fósforo, por otro lado, está relacionado no solo con el desarrollo esquelético, sino con el metabolismo energético (Xiuhua et al., 2016). Las dietas de las aves se basan principalmente en granos de cereales y sus subproductos, las cuales suelen requerir suplementación de fósforo inorgánico debido a las bajas concentraciones y reducida disponibilidad de dicho mineral en tales alimentos (Xiuhua et al., 2016).

Comúnmente se ha empleado la mineralización ósea y el comportamiento productivo como indicadores para determinar el nivel óptimo de calcio y fósforo en la dieta, debido a que la formación de tejido óseo durante su proceso de crecimiento y remodelación requieren la entrada de calcio y fósforo para su formación (Williams et al., 2000). Se han propuesto diversas metodologías para evaluar la mineralización ósea, como el contenido de ceniza, la resistencia a la rotura y el ultrasonido, además de la determinación directa de calcio y fósforo de la tibia (Aguilar et al., 2018). No obstante, dichas metodologías resultan más laboriosas y de mayor costo a otras enfocadas en las características óseas o capacidad para caminar. Al res- 
pecto, la morfometría ósea y la integridad esquelética son técnicas que no requieren laboratorio, considerándose de fácil acceso, permitiendo estimar la mineralización ósea (Kocabagli, 2001; Martínez, 2012); además de considerarse complementarias a las técnicas antes descritas (de Groote y Huyghebaert, 1997).

En la actualidad, se dispone de escasos estudios que hayan evaluado de forma integral la mineralización ósea, integridad esquelética y la morfometría ósea en el fémur, tibia y metatarso del ave, con el fin de tener un panorama más amplio acerca de la salud ósea de las parvadas. Por lo tanto, el objetivo del presente estudio fue determinar el efecto de la relación calcio a fósforo disponible sobre la morfometría y mineralización ósea y la integridad esquelética de pollos de carne.

\section{Materiales y Métodos}

El estudio se llevó a cabo en las instalaciones del Laboratorio de Investigación en Nutrición y Alimentación de Aves (LINAA) de la Universidad Nacional Agraria la Molina (UNALM), Lima, Perú. Se emplearon 60 pollos BB machos de la línea Cobb 500, distribuidos al azar en 12 unidades $(n=12)$ experimentales de cinco aves cada una. Se utilizó una batería con calefacción eléctrica controlada por termostatos y cada unidad experimental contó con un comedero y un bebedero. El programa de iluminación fue de luz continua durante las 24 horas. El tiempo de crianza fue de 21 días.

Los tratamientos fueron: T1, Dieta con relación calcio fósforo baja $1.13(\mathrm{Ca}: 0.50 \%$ - Pd: 044\%); T2, Dieta con relación calcio fósforo media 1.55 (Ca: $0.68 \%$ - Pd: $0.44 \%$ ); T3, Dieta con relación calcio fósforo normal 1.98 (Ca: 0.87\% - Pd: 0.44\%).

Las especificaciones nutricionales estuvieron de acuerdo con la guía nutricional para pollos de carne de la línea Cobb 500
(Cobb-Vantress, 2012). Se formularon tres dietas modificando en cada caso la relación calcio a fósforo disponible (Ca:Pd). Para ello se utilizaron los requerimientos nutricionales promedio de la etapa de inicio y crecimiento. Se empleó una única fase de alimentación durante todo el periodo de evaluación. En el Cuadro 1 se muestra la composición, los valores nutricionales calculados y de los nutrientes de las dietas experimentales.

Las aves se sacrificaron al concluir los 21 días de la fase experimental para realizar las mediciones correspondientes. Se retiraron las patas del cuerpo y se colocaron en agua hirviendo por un lapso de 15 minutos para facilitar la posterior remoción de los tejidos musculares y conectivos (Buckner et al., 1950; Applegate y Lilburn, 2002). Este procedimiento no tiene influencia sobre el contenido mineral ni la densidad del hueso, pero permite remover la grasa de la superficie de los huesos (Almeida Paz et al., 2008). Los tejidos y cartílagos presentes en las zonas articulares se retiraron manualmente siguiendo el protocolo de Baumel et al. (1993) para obtener fémures, tibias y metatarsos limpios. Luego, los huesos fueron secados a temperatura ambiente hasta alcanzar un peso constante.

Se determinó la densidad ósea (peso/ volumen), diámetro de la diáfisis, largo, volumen y peso y porcentaje de cenizas (García y Dale, 2006). Se realizaron dos mediciones para medir el diámetro de la diáfisis: el diámetro laterolateral (DLL) y el diámetro cráneo-caudal (DCC), ambos en el 50\% del largo de la diáfisis (Kocabagli, 2001; Applegate y Lilburn, 2002) y se calculó el promedio de estas dos mediciones. El largo (mm) y el diámetro de la diáfisis $(\mathrm{mm})$ fueron medidos empleando un vernier Uyustools profesional con una capacidad para $15 \mathrm{~cm}$ y con una aproximación de $0.05 \mathrm{~mm}$. Para medir el peso de los huesos (mg) se empleó una balanza analítica con una aproximación de $0.1 \mathrm{mg}$ y para la determinación de las cenizas se utilizó un horno mufla. 
Cuadro 1. Composición, valores nutricionales calculados y nutrientes de las dietas normal y deficiente en fósforo

\begin{tabular}{lccc}
\hline \multirow{2}{*}{ Ingrediente } & \multicolumn{3}{c}{ Dieta } \\
\cline { 2 - 4 } & Ca:Pd (1.13) & Ca:Pd (1.55) & Ca:Pd (1.98) \\
\hline Maíz amarillo molido & 58.15 & 58.15 & 58.15 \\
Torta de soya & 32.52 & 32.52 & 32.52 \\
Aceite crudo de soya & 4.82 & 4.82 & 4.82 \\
Fosfato dicálcico & 1.62 & 1.62 & 1.62 \\
Carbonato de calcio & 0.00 & 0.47 & 0.95 \\
DL-Metionina & 0.26 & 0.26 & 0.26 \\
Lisina-HCL & 0.14 & 0.14 & 0.14 \\
L-Treonina & 0.05 & 0.05 & 0.05 \\
Premezcla vitamina-mineral & 0.12 & 0.12 & 0.12 \\
Cloruro de colina & 0.10 & 0.10 & 0.10 \\
Secuestrante & 0.05 & 0.05 & 0.05 \\
Antioxidante & 0.05 & 0.05 & 0.05 \\
Antibiótico & 0.05 & 0.05 & 0.05 \\
Sal común & 0.47 & 0.47 & 0.47 \\
Material inerte & 1.59 & 1.12 & 0.95 \\
\hline Total & 100.00 & 100.00 & 100.00 \\
\hline Valor nutricional (calculado) & & & \\
E.M., Kcal/kg & 3072 & 3072 & 3072 \\
Proteína total, \% & 20.5 & 20.5 & 20.5 \\
Lisina total, \% & 1.22 & 1.22 & 1.22 \\
Met + Cist total, \% & 0.92 & 0.92 & 0.92 \\
Calcio, \% & 0.50 & 0.68 & 0.87 \\
Fósforo disponible, \% & 0.44 & 0.44 & 0.44 \\
Relación Ca:Pd & 1.13 & 1.55 & 1.98 \\
\hline
\end{tabular}

${ }^{1}$ Laboratorio de Evaluación Nutricional de Alimentos (LENA) - UNALM

Las densidades óseas del fémur, tibia y tarso de las aves se determinaron mediante la volumetría del hueso (Sato, 1995; Zhang et al., 1997; Quarentelli et al., 2007), considerándose como densidad ósea a la masa de material orgánico e inorgánico en el hueso por unidad de volumen de este (Rath et al., 2000). Se calculó dividiendo el peso del hueso $(\mathrm{mg})$ entre el volumen $\left(\mathrm{cm}^{3}\right)$. Para la determinación del volumen de los huesos se emplearon probetas graduadas con capacidad de 10 y $25 \mathrm{~cm}^{3}$ y con una aproximación de $0.2 \mathrm{~cm}^{3}$ y $0.5 \mathrm{~cm}^{3}$, respectivamente.
En el día 21 se evaluó, de manera cualitativa, la capacidad para caminar del ave empleando una modificación del score propuesto por Kestin et al. (1992): 1, incapacidad para sostenerse sobre sus patas; 2, gran dificultad para caminar; 3 , lesión evidente; 4 , anormalidad definida para caminar; 5 , defectos leves; 6, normal. Además, se evaluó la degeneración ósea del fémur en las aves sacrificadas empleando el Índice de Degeneración Femoral (Almeida Paz et al., 2008) con la siguiente escala: 1, hueso sin lesión; 2, el cartílago está ausente en la cabeza femoral, 
pero el hueso está intacto; 3 , la cabeza femoral no tiene el cartílago y está parcialmente rota; 4, la cabeza femoral está considerablemente dañada, pero su contorno está aún visible; 5 , la cabeza del fémur está completamente rota y no es posible reconocer su contorno. El contenido de ceniza en la tibia fue determinado empleando el método oficial 942.05 de la AOAC (Thiex et al., 2012).

Se empleó un diseño completamente al azar con tres tratamientos y cuatro repeticiones por tratamiento. El análisis de varianza se llevó a cabo usando el programa Statistical Analysis System (SAS, 2000). La comparación entre medias se determinó mediante la prueba de Duncan.

\section{Resultados y Discusión}

El efecto de la relación Ca:Pd sobre las características morfométricas de los huesos evaluados se presenta en el Cuadro 2. Los tratamientos dietarios no afectaron significativamente el largo de los tres huesos de la pierna; resultados que concuerdan con Da silva et al. (2003), quienes no encontraron diferencias significativas en el largo de la tibia cuando se modificó la relación Ca:Pd de 1.70 a 2.27. En un estudio con niveles de calcio y fósforo siguiendo los requerimientos de la línea genética, Applegate y Lilburn (2002) reportaron longitudes de fémur y tibia de 49.00 y $67.20 \mathrm{~mm}$, respectivamente; valores que concuerdan con los obtenidos en el presente estudio. Por su parte, tras un estudio sobre el desarrollo del fémur, Ribeiro et al. (2011) reportaron un largo del fémur de $54.36 \mathrm{~mm}$ en pollos de 22 días utilizando una dieta con niveles adecuados de calcio y fósforo recomendados por la línea genética. En las dos investigaciones anteriores se presentaron promedios de longitudes óseas que guardan relación con los promedios obtenidos del largo del fémur y de la tibia en el presente estudio.

La tibia, particularmente al final de la epífisis, ha sido ampliamente estudiada debido a la sensibilidad celular a numerosas defi- ciencias en la dieta (Leach y Lilburn, 1992) y a su alta tasa de crecimiento en comparación con otros huesos largos (Buckner et al., 1950); además, la tibia es el hueso más largo y el que tiene la tasa de crecimiento más alta en comparación con el fémur y el metatarso. Se ha establecido que el largo de la tibia está estrechamente relacionado al peso del animal y, este a su vez, está influenciado por el sexo; es decir que los machos al tener un mayor peso tienen huesos de la pierna más largos (Buckner et al., 1950; Bond et al., 1991). Por su parte, Williams et al. (2010) compararon la morfometría de la tibia de pollos de engorde de dos líneas Ross, una de lento y otra de rápido crecimiento, encontrando diferencias significativas en el largo de la tibia entre las líneas genéticas, concluyendo que la longitud de este hueso ha disminuido significativamente con el paso del tiempo por el proceso de selección.

No se encontraron diferencias significativas en el ancho de los tres huesos de la pierna (Cuadro 2). En estudios recientes, Marques et al. (2013) tampoco encontraron diferencias significativas en el ancho de la tibia en pollos de 21 días de edad con diversos niveles de vitamina $\mathrm{D}$, y los valores reportados de 6.13 y $5.50 \mathrm{~mm}$ para ancho de fémur y tibia, respectivamente, son relativamente similares a los del presente estudio (7.06 y $6.00 \mathrm{~mm}$ para fémur y tibia, respectivamente); diferencias que se pueden atribuir al lugar de medición en la diáfisis de los huesos evaluados. Valores numéricos similares del ancho de los huesos fueron encontrados por Applegate y Lilburn (2002), cuyos valores de ancho de fémur de $5.86 \mathrm{~mm}$ y de ancho de tibia de $5.47 \mathrm{~mm}$. Rutten et al. (2002) reportaron valores de $5.45 \mathrm{~mm}$ de ancho de tibia con una dieta conteniendo $\mathrm{Ca}$ y $\mathrm{P}$ en una relación adecuada.

En otros estudios, Ribeiro et al. (2011) reportaron valores del ancho del fémur en pollos de 22 días de $5.35 \mathrm{~mm}$ usando una dieta con balance adecuado de calcio y fósforo. Por su parte, Williams et al. (2000) encontraron diferencias en el ancho del hueso de la 
tibia entre dos líneas genéticas (lento crecimiento; $5.0 \mathrm{~m}$; rápido crecimiento; $3.8 \mathrm{~mm}$ ), indicando una reducción significativa del ancho de este hueso con el avance genético.

El volumen de los tres huesos de la pierna no fue afectado por las relaciones de Ca:Pd utilizadas en el presente estudio (Cuadro 2). Al respecto, Quarantelli et al. (2007) no encontraron diferencias significativas en el volumen de la tibia cuando evaluaron niveles de biotina. Es importante indicar que en el presente experimento el volumen de la tibia fue mayor que el volumen del fémur, y este a su vez resultó mayor que el volumen del metatarso, aunque sin diferencias significativas. Esta tendencia que se observó en todas las relaciones de $\mathrm{Ca}: \mathrm{Pd}$ utilizadas.

El peso de los tres huesos no presentó diferencias significativas entre las dietas experimentales (Cuadro 2); sin embargo, el peso del fémur y el de la tibia presentaron en el análisis de varianza un valor de $\mathrm{P}$ igual a 0.0739 y 0.0554 , respectivamente; indicando una posible influencia marginal de los tratamientos sobre estas dos variables. De forma coincidente, da Silva et al. (2003) observaron en pollos de 21 días de edad que el peso de la tibia disminuye significativamente cuando se modifica la relación Ca:Pd desde 1.70 hasta 2.27. Valores similares para el peso del fémur $(1670 \mathrm{mg}$ ) y de la tibia $(2360 \mathrm{mg})$ fueron reportados por Applegate y Lilburn (2002). Por su parte, Ribeiro et al. (2011) reportaron pesos de $4640 \mathrm{mg}$ para el fémur de pollos de 22 días, alimentados con una dieta balanceada según la línea genética, peso que no concuerda con los $1599.50 \mathrm{mg}$ encontrados en la presente investigación, debido posiblemente al método empleado para el procesamiento del hueso.

La densidad del hueso es un importante indicador para conocer el estado de salud del esqueleto (Almeida Paz et al., 2008) y puede ser medida de forma directa, como en el presente estudio, o empleando métodos que permiten su estimación, como la absorciometría de rayos-X de energía dual (Angel et al., 2006). En el presente estudio, la relación $\mathrm{Ca}: P d$ de 1.13 fue la única que afectó la den$\operatorname{sidad}(\mathrm{p}<0.05$; Cuadro 2). Al respecto, da Silva et al. (2003) indican que la densidad de la tibia disminuye significativamente ante un cambio en la relación Ca:Pd de 1.70 a 2.27 en pollos de la línea Cobb 500; sin embargo, ante el mismo cambio en la relación $\mathrm{Ca}: \mathrm{Pd}$ los pollos de la línea Avian Farms no mostraron diferencias significativas, presumiéndose que estos últimos presentan un menor requerimiento de minerales o presentan una baja tasa de crecimiento inicial.

En el Cuadro 3 se observa la respuesta de la integridad esquelética. La capacidad para caminar se ve afectada cuando la relación $\mathrm{Ca}: \mathrm{Pd}$ es 1.13. La patología conocida como necrosis de cabeza femoral no presentó diferencias significativas entre tratamientos, y en ninguno se presentó casos severos de esta patología.

Thorp y Waddington (1997) mostraron los beneficios potenciales de la combinación entre las patologías óseas como complemento al análisis de contenido mineral. Sus resultados muestran un incremento del contenido de ceniza en tibia en las aves que presentaron necrosis de cabeza femoral (condición que disminuye cualquier parámetro productivo); por lo tanto, no necesariamente un indicativo de una adecuada integridad esquelética es el contenido de ceniza en el hueso, ya que esta variable necesita ser complementada con indicadores de integridad esquelética.

La incidencia de problemas de patas en pollos de rápido crecimiento puede ser afectada por el contenido mineral de la dieta (Nelson et al., 1990). Así, un nivel adecuado de calcio reduce significativamente los problemas a nivel de patas (Thorp y Waddington, 1997). Por su parte, Takita (1998) concluyó que los pollos de engorde que presentaron un rápido crecimiento durante la fase inicial son más propensos a desarrollar anormalidades del sistema locomotor y pollos con una baja en la relación $\mathrm{Ca}: \mathrm{Pd}$ presentaron evidencia 
Cuadro 2. Efecto de la relación Ca:Pd sobre las características morfométricas de huesos de pollos parrilleros sometidos a dietas con tres niveles de la relación Ca:Pd

\begin{tabular}{lccc}
\hline \multirow{2}{*}{ Medición } & \multicolumn{3}{c}{ Relación Ca:Pd } \\
\cline { 2 - 4 } & \multicolumn{1}{c}{$\mathrm{T} 1^{1}$} & $\mathrm{~T} 2$ & $\mathrm{~T} 3$ \\
\hline Largo del fémur, mm & $48.86^{\mathrm{a}}$ & $49.63^{\mathrm{a}}$ & $50.01^{\mathrm{a}}$ \\
Largo de la tibia, mm & $63.98^{\mathrm{a}}$ & $65.10^{\mathrm{a}}$ & $66.04^{\mathrm{a}}$ \\
Largo del metatarso, mm & $47.16^{\mathrm{a}}$ & $48.28^{\mathrm{a}}$ & $48.43^{\mathrm{a}}$ \\
Ancho del fémur, mm & $6.91^{\mathrm{a}}$ & $7.09^{\mathrm{a}}$ & $7.18^{\mathrm{a}}$ \\
Ancho de la tibia, mm & $5.79^{\mathrm{a}}$ & $5.97^{\mathrm{a}}$ & $5.85^{\mathrm{a}}$ \\
Ancho del metatarso, mm & $6.53^{\mathrm{a}}$ & $6.37^{\mathrm{a}}$ & $6.25^{\mathrm{a}}$ \\
Volumen del fémur, $\mathrm{cm}^{3}$ & $2.30^{\mathrm{a}}$ & $2.40^{\mathrm{a}}$ & $2.44^{\mathrm{a}}$ \\
Volumen de la tibia, $\mathrm{cm}^{3}$ & $2.73^{\mathrm{a}}$ & $3.02^{\mathrm{a}}$ & $2.90^{\mathrm{a}}$ \\
Volumen del metatarso, $\mathrm{cm}^{3}$ & $1.97^{\mathrm{a}}$ & $1.98^{\mathrm{a}}$ & $2.02^{\mathrm{a}}$ \\
Peso del fémur, mg & $1474.00^{\mathrm{a}}$ & $1625.25^{\mathrm{a}}$ & $1699.25^{\mathrm{a}}$ \\
Peso de la tibia, mg & $1908.50^{\mathrm{a}}$ & $2186.25^{\mathrm{a}}$ & $2243.38^{\mathrm{a}}$ \\
Peso del metatarso, $\mathrm{mg}$ & $1329.50^{\mathrm{a}}$ & $1433.63^{\mathrm{a}}$ & $1422.75^{\mathrm{a}}$ \\
Densidad del fémur, mg/cm $\mathrm{cm}^{3}$ & $645.37^{\mathrm{a}}$ & $679.98^{\mathrm{a}}$ & $697.34^{\mathrm{a}}$ \\
Densidad de la tibia, $\mathrm{mg} / \mathrm{cm}^{3}$ & $708.18^{\mathrm{b}}$ & $730.75^{\mathrm{ab}}$ & $783.16^{\mathrm{a}}$ \\
Densidad del metatarso, $\mathrm{mg} / \mathrm{cm}^{3}$ & $675.74^{\mathrm{a}}$ & $726.89^{\mathrm{a}}$ & $710.05^{\mathrm{a}}$ \\
\hline
\end{tabular}

a,b,c Superíndices diferentes dentro de filas indican diferencia estadística $(p<0.05)$

${ }^{1}$ Tratamientos: T1: relación Ca:Pd 1.13; T2: relación Ca:Pd 1.55; T3: relación Ca:Pd 1.98

Cuadro 3. Efecto de la relación Ca:Pd sobre la integridad esquelética de pollos parrilleros de 21 días

\begin{tabular}{|c|c|c|c|}
\hline \multirow{2}{*}{ Medición } & \multicolumn{3}{|c|}{ Relación Ca:Pd } \\
\hline & $\mathrm{T} 1^{1}$ & $\mathrm{~T} 2$ & T3 \\
\hline $\begin{array}{l}\text { Capacidad para } \\
\text { caminar }\end{array}$ & $4.20^{\mathrm{b}}$ & $4.91^{\mathrm{ab}}$ & $5.25^{\mathrm{a}}$ \\
\hline $\begin{array}{l}\text { Necrosis de } \\
\text { cabeza femoral }\end{array}$ & $1.00^{\mathrm{a}}$ & $1.11^{\mathrm{a}}$ & $1.00^{\mathrm{a}}$ \\
\hline
\end{tabular}

histológica de raquitismo. La causa probable sería la alteración de la estructura del cristal formador de la hidroxiapatita, debilitando el hueso y contribuyendo a la aparición de fracturas óseas.
El contenido de ceniza de la tibia es la variable más usada en estudios de minerales que involucren al calcio y al fósforo (Pintar et al., 2004; Acosta et al., 2009; Chung et al., 2013; Walk et al., 2014). En este estudio, el contenido de ceniza en la tibia fue de 41.83, 44.12 y $45.51 \%$ para $\mathrm{T} 1, \mathrm{~T} 2$ y $\mathrm{T} 3$, respectivamente, siendo los tres valores significativamente diferentes entre sí $(\mathrm{p}<0.01)$, confirmando que el contenido de ceniza en tibia es altamente sensible a la variación en la relación Ca:Pd. Yan et al. (2005) modificaron la relación Ca:Pd desde 1.30 hasta 2.50 encontrando valores de ceniza en tibia en un rango de 29.94 a $41.32 \%$ con las relaciones $\mathrm{Ca}: \mathrm{Pd}$ de 1.30 y 2.50 , respectivamente. En diversos estudios se ha utilizado la máxima mineralización como un criterio para determinar el requerimiento de fósforo y calcio de las aves; sin embargo, lo razonable es encontrar una relación $\mathrm{Ca}: \mathrm{Pd}$ que produzca un máximo crecimiento, una máxima transfor- 
mación del alimento y una adecuada, pero no necesariamente máxima, mineralización ósea (Abudabos, 2012).

Driver et al. (2005) comprobaron que la relación calcio a fósforo disponible de 1.39 fue la que mostró la mayor respuesta productiva en función del peso; sin embargo, el máximo contenido de ceniza en tibia se consiguió con la relación calcio a fósforo de 2.0, motivo por el cual los autores sugieren que existen diferentes requerimientos de calcio para la máxima respuesta productiva y para una máxima deposición de ceniza en tibia. Bar et al. (2003) destacan la capacidad de adaptación de los pollos de engorde modernos a bajos niveles de calcio y fósforo en la dieta. Estos autores concluyen que el requerimiento de fósforo es similar para obtener la máxima respuesta productiva y la máxima mineralización, pero el requerimiento de calcio es menor para obtener la máxima respuesta productiva en comparación con un nivel mayor de calcio para obtener una mayor mineralización en cuanto al contenido de ceniza en tibia y, por lo tanto, al elegir el nivel de calcio adecuado se debe determinar con antelación si el objetivo es una máxima mineralización o una máxima respuesta productiva.

\section{ConClusiones}

- La densidad del hueso tibia es la característica ósea de morfometría más influenciada por cambios en la relación Ca:Pd.

- La integridad esquelética evaluada a través de la capacidad para caminar está influenciada con relaciones Ca:Pd de 1.13.

- El contenido de ceniza en tibia fue la variable más sensible a cambios en relación $\mathrm{Ca}: \mathrm{Pd}$.

\section{Literatura Citada}

1. Abudabos AM. 2012. Optimal dietary phosphorus for broiler performance,- bone integrity and reduction of phosphorus excretion. Asian J Anim Vet Adv 7: 288-298. doi: 10.3923/ajava.2012.288.298.

2. Acosta A, Lon-Wo E, Cárdenas M, Febles M, Dieppa O, Almeida M. 2009. Determinación de la biodisponibilidad relativa del fósforo en la fosforita del yacimiento Trinidad de Guedes, mediante pruebas de crecimiento y mineralización ósea en pollos y gallinas ponedoras. Rev Cubana Cienc Agríc 43: 55-59.

3. Aguilar J, Zea O, Vílchez C. 2018. Rendimiento productivo e integridad ósea de pollos de carne en respuesta a suplementación dietaria con cuatro fuentes de fitasa comercial. Rev Inv Vet Peru 29: 169-179. doi: 10.15381/rivep.v29i1.14078

4. Almeida ICL, Mendes AA, Balog A, Vulcano LC, Ballarin AW, Almeida ICL, et al. 2008. Study on the bone mineral density of broiler suffering femoral joint degenerative lesions. Braz J Poult Sci 10: 103-108. doi: 10.1590/ S1516-635X2008000200005

5. Angel R, Saylor WW, Mitchell AD, Powers W, Applegate TJ. 2006. Effect of dietary phosphorus, phytase, and 25hydroxycholecalciferol on broiler chicken bone mineralization, litter phosphorus, and processing yields. Poult Sci 85: 12001211. doi: $10.1093 / \mathrm{ps} / 85.7 .1200$.

6. Applegate TJ, Lilburn MS. 2002. Growth of the femur and tibia of a commercial broiler line. Poult Sci 81: 1289-1294. doi: 10.1093/ps/81.9.1289

7. Bar A, Shinder D, Yosefi S, Vax E, Plavnik I. 2003. Metabolism and requirements for calcium and phosphorus in the fast-growing chicken as affected by age. Brit J Nutr 89: 51-60. doi: 10.1079/BJN2002757

8. Baumel JJ, Witmer LM, King AS, Breazile JE, Evans HE, Vanden Berge JC. 1993. Handbook of avian anatomy: Nomina anatomica avium. $2^{\text {nd }}$ ed. Publication N. ${ }^{\circ} 23$. Massachusetts, USA: Nuttall Ornithological Club. 401 p. 
9. Bond PL, Sullivan TW, Douglas JH, Robeson LG. 1991. Influence of age, sex, and method of rearing on tibia length and mineral deposition in broilers. Poult Sci 70: 1936-1942. doi: 10.3382/ ps.0701936

10. Buckner GD, Insko WM, Harms A, Faull E. 1950. The comparative rates of growth and calcification of the femur, tibia and metatarsus bones of the male and female New Hampshire chicken having straight keel. Poult Sci 29: 332335. doi: $10.3382 /$ ps. 0290332

11. Chung TK, Rutherfurd SM, Thomas DV, Moughan PJ. 2013. Effect of two microbial phytases on mineral availability and retention and bone mineral density in low-phosphorus diets for broilers. Brit Poult Sci 54: 362-373. doi: 10.1080/ 00071668.2013 .783902

12. Cobb-Vantress. 2012. Guía de manejo del pollo de engorde. [Internet]. Disponible en: https://cobbstorage.blob.core.windows.net/guides/a71b8bc0bbd4-11e6-bd5d-55bb08833e29.pdf

13. da Silva CS; Baraldi SM, Francelino L, Quirino MF, Mack JO, Roque AI. 2003. Bone development of broiler chickens fed diets with different amino acid and calcium levels during the starter phase. Int J Morphol 21: 101-106. doi: $10.4067 / \mathrm{S} 0717-95022003000200001$

14. de Groote G, Huyghebaert G. 1997. The bio-availability of phosphorus from feed phosphates for broilers as influenced by bio-assay method, dietary Ca-level and feed form. Anim Feed Sci Tech 64: 329340.

15. Driver JP, Pesti GM, Bakalli RI, Edwards Jr HM. 2005. Calcium requirements of the modern broiler chicken as influenced by dietary protein and age. Poult Sci 84: 1629-1639. doi: 10.1093/ps/84.10.1629

16. García AR, Dale NM. 2006. Foot ash as a means of quantifying bone mineralization in chicks. J Appl Poult Res 15:103-109. doi: 10.1093/japr/15.1.103
17. Han HC, Qu HX, Wang, JG, Chen GH, Yan YF, Zhang JL, Hu FM, et al. 2015. Comparison of the growth and mineralization of the femur, tibia, and metatarsus of broiler chicks. Braz J Poult Sci 17: 333-340. doi: 10.1590/1516635X1703333-340

18. Kayode Z, Omolara M, Henry O. 2016. Effect of strain and age on bone integrity of commercial broiler chickens. Biotechnol Anim Husb 32: 195-203. doi: 10.2298/BAH1602195

19. Kestin SC, Knowles TG, Tinch AE; Gregory NG 1992. Prevalence of leg weakness in broiler chickens and its relationship with genotype. Vet Rec 131: 190-194. doi: 10.1136/vr.131.9.190

20. Kocabagli N. 2001. The effect of dietary phytase supplementation at different levels on tibial bone characteristics and strength in broilers. Turk J Vet Anim Sci 25: 797-802.

21. Leach RM, Lilburn MS. 1992. Current knowledge on the etiology of tibial dyschondroplasia in the avian species. Poult Sci 4: 57-65.

22. Marques A, Murakami A, do Amaral C, Ospina I, Picoli K, Mangili M. 2013. Use of vitamin $D_{3}$ and its metabolites in broiler chicken feed on performance, bone parameters and meat quality. Asian Austral J Anim 26: 408415. doi: 10.5713/ajas.2012.12455

23. Martínez DA. 2012. Evaluación de un producto a base de aceite esencial de orégano sobre la integridad intestinal, la capacidad de absorción de nutrientes y el comportamiento productivo de pollos de carne. Tesis de Magíster. Lima: Univ. Nacional Agraria La Molina. $268 \mathrm{p}$.

24. Nelson TS, Kirby LK, Johnson ZB. 1990. Effect of minerals on the incidence of leg abnormalities in growing broiler chickens. Nut Res 10: 525-533.

25. Pintar J, Homen B, Gazic K, Grbesa D, Sikiric M, Cerny T. 2004. Effects of supplemental phytase on performance and tibia ash of broiler fed different cereals based diets. Czech J Anim Sci 49: 542-548. 
26. Quarantelli A, Cacchioli A, Romanelli S, Righi F, Alpigiani I, Gabbi C. 2007. Effects of different levels of dietary biotin on the performance and bone structure of broilers. Ital J Anim Sci 6: 5-17. doi: 10.4081/ijas.2007.5

27. Rath NC, Huff GR, Huff WW, Balog JM. 2000. Factors regulating bone maturity and strength in poultry. Poult Sci 79: 1024-1032. doi: 10.1093/ps/79.7.1024

28. Ribeiro F, Martínez S, Augusto L, Barbosa J, Morais A, Rita M, Amoroso L. 2011. Determination of broiler femur parameters at different growth phases. Int J Poultry Sci 10: 849-853. doi: 10.3923/ijps.2011.849.853

29. Rutten M, Leterrier Cu, Constantin P, Reiter K, Bessei W. 2002. Bone development and activity in chickens: response to reduced weight-load on legs. Anim Res 51: 327-336.

30. SAS Institute. 2000. The SAS System for Windows. User's Guide Statistics v8. SAS Institute, Inc., Cary, USA.

31. Sato M. 1995. Comparative X-ray densitometry of bones from ovariectomized rats. Bone 17: 157-162. doi: 10.1016/8756-3282(95)00288-O

32. Takita TS. 1998. Efeito do genotipo, do ambiente e da interacao genotipo $\mathrm{x}$ ambiente na incidencia de discondroplasia tibial em frangos de corte machos. Tese de Mestrado. Brasil: Univ. Estadual Paulista. $41 \mathrm{p}$.

33. Thiex N, Novotny L, Crawford A. 2012. Determination of ash in animal feed: AOAC official method 942.05 revisited. J AOAC Int 95: 1392-1397. doi: 10.5740/jaoacint.12-129

34. Thorp BH, Waddington D. 1997. Relationships between the bone pathologies, ash and mineral content of long bones in 35-day-old broiler chickens. Res Vet Sci 62: 67-73. doi: 10.1016/S0034-5288(97)90183-1

35. Yan F, Angel R, Ashwell C, Mitchell A, Christman M. 2005. Evaluation of the broiler's ability to adapt to an early moderate deficiency of phosphorus and calcium. Poult Sci 84: 1232-1241. doi: $10.1093 / \mathrm{ps} / 84.8 .1232$

36. Walk CL, Santos TT, Bedford MR. 2014. Influence of superdoses of a novel microbial phytase on growth performance, tibia ash, and gizzard phytate and inositol in young broilers. Poult Sci 93: 1172-1177. doi: 10.3382/ps.2013-03571

37. Williams B, Waddington D, Solomon S, Thorp B, Farquharson C. 2010. Skeletal development in the meat-type chicken. Brit Poultry Sci 41: 141-149. doi: 10.1080/713654918.

38. Li X, Zhang D, Yang TY, Bryden WL. 2016. Phosphorus bioavailability: a key aspect for conserving this critical animal feed resource with reference to broiler nutrition. Agriculture 6: 1-15. doi: 10.3390/agriculture6020025

39. Zhang X, Liu G, McDaniel G.R, Roland DA. 1997. Responses of broiler lines selected for tibial dyschondroplasia incidence to supplementary 25hydroxycholecalciferol. J Appl Poultry Res 6: 410-416. doi: 10.1093/japr/6.4.410 